\title{
Video Article \\ Cochlear Implant Surgery and Electrically-evoked Auditory Brainstem Response Recordings in C57BL/6 Mice
}

\author{
Charlotte Amalie Navntoft ${ }^{1,2}$, Jeremy Marozeau ${ }^{1}$, Tania Rinaldi Barkat ${ }^{2}$ \\ ${ }^{1}$ Hearing Systems Group, Department of Electrical Engineering, Technical University of Denmark \\ ${ }^{2}$ Brain and Sound Lab, Department of Biomedicine, Basel University \\ Correspondence to: Charlotte Amalie Navntoft at charlotte.navntoft@unibas.ch \\ URL: https://www.jove.com/video/58073 \\ DOI: doi: $10.3791 / 58073$
}

Keywords: Neuroscience, Issue 143, Cochlear implant, mouse model, auditory nerve, electrically auditory brainstem response, C57B mouse, ototoxic deafening, neomycin, round window

Date Published: 1/9/2019

Citation: Navntoft, C.A., Marozeau, J., Barkat, T.R. Cochlear Implant Surgery and Electrically-evoked Auditory Brainstem Response Recordings in C57BL/6 Mice. J. Vis. Exp. (143), e58073, doi:10.3791/58073 (2019).

\section{Abstract}

Cochlear implants $(\mathrm{Cls})$ are neuroprosthetic devices that can provide a sense of hearing to deaf people. However, a $\mathrm{Cl}$ cannot restore all aspects of hearing. Improvement of the implant technology is needed if $\mathrm{Cl}$ users are to perceive music and perform in more natural environments, such as hearing out a voice with competing talkers, reflections, and other sounds. Such improvement requires experimental animals to better understand the mechanisms of electric stimulation in the cochlea and its responses in the whole auditory system. The mouse is an increasingly attractive model due to the many genetic models available. However, the limited use of this species as a Cl model is mainly due to the difficulty of implanting small electrode arrays. More details about the surgical procedure are therefore of great interest to expand the use of mice in $\mathrm{Cl}$ research.

In this report, we describe in detail the protocol for acute deafening and cochlear implantation of an electrode array in the C57BL/6 mouse strain. We demonstrate the functional efficacy of this procedure with electrically-evoked auditory brainstem response (eABR) and show examples of facial nerve stimulation. Finally, we also discuss the importance of including a deafening procedure when using a normally hearing animal. This mouse model provides a powerful opportunity to study genetic and neurobiological mechanisms that would be of relevance for $\mathrm{Cl}$ users.

\section{Video Link}

The video component of this article can be found at https://www.jove.com/video/58073/

\section{Introduction}

Cochlear implants $(\mathrm{Cls})$ are electronic devices that can provide a sense of hearing to people with severe and profound hearing loss. It uses electrodes surgically implanted in the cochlea of the inner ear to directly stimulate the auditory nerve. To date, the $\mathrm{Cl}$ is the most successful sensory prosthesis and has helped more than 600,000 people worldwide ${ }^{1}$. However, the device has shortcomings. First, the benefits provided by the device vary greatly among recipients. Second, speech in noisy environments and music are still poorly perceived by most $\mathrm{Cl}$ users.

For many years, animal models have been used to better understand these issues in $\mathrm{Cl}$ research and to continuously improve safety and efficacy of the devices. The models have given valuable insight into several phenomena, such as plastic changes in the brain taking place following $\mathrm{Cl}$ implantation ${ }^{2}$, the effect of applying gene therapy to preserve residual hearing ${ }^{3}$, and biophysical properties of the electrically stimulated auditory nerve ${ }^{4}$, among many other examples.

Mice are a powerful model organism due to the large availability of genetic models of deafness. Other advantages include the ability to manipulate the mouse genome (e.g., via the CRISPR-Cas system), the opportunity to use advanced imaging techniques to study mechanisms, particularly in the brain, the high reproduction rate, rapid development and easy breeding and handling. The main technical challenges in performing $\mathrm{Cl}$ surgeries in mice are the small size of the cochlea and the presence of a large stapedial artery (SA). The SA usually disappears during embryonic development in humans but persists throughout life in a number of rodents, including mice, rats, and gerbils. The SA runs below the round window niche, which complicates access to the cochlea and increases surgical risk.

Previous studies have shown the feasibility of $\mathrm{Cl}$ implantation in mice ${ }^{5,6,7}$. Irving et al. demonstrated that chronic intracochlear electrical stimulation can be achieved for up to one month. Acute stimulation was also performed but the recordings were not presented. They showed that cauterizing the stapedial artery had no significant effect on the hearing threshold or the number of spiral ganglion neurons and that topical application of the aminoglycoside neomycin, an ototoxic drug, was an effective deafening procedure in mice ${ }^{5}$. Soken et al. described a modified dorsal approach to the mouse cochlea through the round window to better preserve hearing status ${ }^{6}$. Following insertion of a platinum-iridium wire, substantial residual hearing was observed with an increased auditory brainstem response (ABR) threshold of $28 \mathrm{~dB}$. Otoacoustic emissions (OAE) were lost in animals with large ABR threshold shifts ${ }^{6}$. Mistry et al. tested the functional and histopathological effects of implantation in the 
absence of electric stimulation ${ }^{7}$. Even though hearing was preserved in both 3 and 6 months-old implanted mice at low frequencies, implantation resulted in fibrosis-like tissue around the implant and osteoneogenesis around the bullostomy ${ }^{7}$.

In short, out of the three studies on Cls in mice, only one demonstrates functional recording of $\mathrm{Cl}$ stimulation. Irving and colleagues performed both acute and chronic eABR recordings but only showed data from chronic Cl stimulation ${ }^{5}$. However, the chronic model with a fully implantable device developed by Irving et al. is technically challenging. It is not yet known if acute $\mathrm{Cl}$ stimulation, both less challenging and faster, can achieve similar results.

$\mathrm{Cls}$ are used by people with severe and profound hearing loss who no longer benefit from hearing aids. Animal models for $\mathrm{Cl}$ users should therefore include a deafening procedure when normally hearing animals are used. Another reason to deafen hearing animals is that the electrical stimulation of a deaf or hearing cochlea produces different neural responses ${ }^{4,8,9,10,11,12}$. Electrical stimulation of a deaf cochlea directly activates the auditory nerve fibers and generates an electroneural response $(\alpha)$. It is characterized by short latency and a small dynamic range in the periphery ${ }^{8,10}$. On the other hand, electrical stimulation of a hearing cochlea also excites the hair cells in an electrophonic response $(\beta)$ that is characterized by longer latencies and larger dynamic range $e^{4,11}$. The electrophonic response is attributed to normal excitation of nerve fibers by inner hair cells, electrically induced contraction of outer hair cells, and generation of a travelling wave ${ }^{4}$. Electroneural and electrophonic responses also result in two different activity patterns in the central nervous system ${ }^{9}$. Sato et al. recorded midbrain neurons of a $\mathrm{Cl}$ implanted guinea pig before and after deafening with neomycin, which eliminates the electrophonic contribution. They showed that the slope of the ratelevel function was steeper and firing rates higher in the deafened condition compared to the hearing condition ${ }^{9}$. Therefore, depending on the research question stated, it is important to consider including deafening to separate electrophonic and electroneural responses upon electric stimulation of the auditory nerve.

Here, we describe the procedure for acute deafening and the cochlear implantation of an electrode array in a mouse as well as the functional recording of intracochlear electric stimulation with electrically-evoked auditory brainstem response (eABR).

\section{Protocol}

All procedures were carried out according to Basel University, Switzerland, animal care and guidelines. They were licensed by the Veterinary Office of the Canton of Basel, Switzerland.

NOTE: C57BL/6 adult mice, aged 8-12 weeks (weight 20-30 g), were used in this study.

The left ear is used as the experimental ear. The right ear serves as an intra-animal control and is not surgically altered.

\section{Preoperative Procedures}

1. Anaesthetize the animal $30 \mathrm{~min}$ prior to surgery via intra-peritoneal (i.p.) injection of ketamine/xylazine (80 $\mathrm{mg} / \mathrm{kg} \mathrm{ketamine,} 16 \mathrm{mg} /$ kg xylazine, i.p., volume injected at $10 \mu \mathrm{L} / \mathrm{g}$ body weight).

1. Supplement anesthesia as necessary, as judged by a positive pedal and palpebral (toe-pinch) reflex and movement of the whiskers, with a lower dose of ketamine ( $45 \mathrm{mg} / \mathrm{kg}$, i.p., injected at $10 \mu \mathrm{L} / \mathrm{g}$ body weight). Agents and dose regimes can be substituted per institutional guidelines.

Notes: In general, the animal will need an injection every 45-60 min with this agent and dose regime. The average time from initial incision to closure around the implanted electrode array is typically 1-1.5 hours.

2. Check for full sedation of the animal marked by a regular breathing rate and a lack of toe-pinch reflexes. Maintain this level of anesthesia.

3. Maintain the animal's body temperature at $36.6^{\circ} \mathrm{C}$ with a closed-loop heating pad. Apply eye ointment to avoid dehydration of the cornea. This will also suppress the animal's blink reflex, which can add noise to the recoding.

4. Administer local analgesic via subcutaneous injection (s.c.) of bupivacaine/Lidocaine $(0.1 \mathrm{mg} / \mathrm{mL}$ bupivacaine and $0.4 \mathrm{mg} / \mathrm{mL}$ lidocaine, 0.1 $\mathrm{mL}$ administered s.c.) along the intended incision line to minimize any surgical discomfort. Agents and dose regimes can be substituted per institutional guidelines.

5. Administer the muscarinic antagonist atropine (atropinesulfate amino, $0.1 \mathrm{mg} / \mathrm{mL}, 20 \mu \mathrm{L}$ administered s.c., dissolved in PBS) in the neck to reduce mucus secretion and to facilitate breathing. Agents and dose regimes can be substituted per institutional guidelines.

\section{Pre-deafening Acoustic Auditory Brainstem Response (aABR)}

NOTE: AABR is used to measure the status of hearing before and after deafening. Testing is performed on the left ear and in a soundproof electrically shielded booth. We recommend to test and later implant the left ear for a right-handed person. Further details on ABR in mice can be found in ${ }^{13,14}$. Tucker Davis Technologies (TDT) hardware and software (BioSig) are used to record ABR but other systems can be used.

1. Block the contralateral (right) ear with acoustic foam to isolate the ABR response from the ipsilateral (left) ear. Put the foam in a $1 \mathrm{~mL}$ syringe and inject it into the right ear canal of the mouse to cover the whole ear canal with foam $(0.1-0.2 \mathrm{~mL}$ of foam). Make sure the syringe seals closely to the ear so that the foam gets all the way into the ear canal.

2. Place the speaker $10 \mathrm{~cm}$ from the left ear. NOTE: The speaker for this setup was calibrated using a PCB microphone as described in refefence ${ }^{15}$.

3. Clean the ABR electrodes with $70 \%$ ethanol solution. Place the electrodes under the skin: active (Ch1) on the vertex, reference (-) below the pinna of the ipsilateral ear, and ground in the hind leg (Figure 1).

4. Connect the head-stage and pre-amplifier to the auditory processor via the optic fiber port.

5. Check the impedance of the active and reference electrode.

1. If the impedance is over $3 \mathrm{Ohm}$, re-arrange them and re-take the measurement. The best recordings are obtained when the electrodes have the same impedance. Close the sound-proof booth. 
6. Present click stimulation and record $A B R$ in a free-field condition with a complex auditory processor and software. Standardize the click stimulus in the software: $0.1 \mathrm{~ms}$ single-channel monophasic clicks are presented at $21 \mathrm{~Hz}$; Click level decreases from $90 \mathrm{~dB}$ SPL to $10 \mathrm{~dB}$ $\mathrm{SPL}$ in $10 \mathrm{~dB}$ steps; $10 \mathrm{~ms}$ recording window. Average a total of 512 responses at each $\mathrm{dB}$ level.

7. Apply a $2,000 \mathrm{~Hz}$ lowpass filter and a $300 \mathrm{~Hz}$ highpass filter offline to reduce noise in the recording using a custom-made Matlab script

8. Determine the ABR threshold as the lowest $d B$ level with a recognizable ABR wave response (Figure 2, Figure 3).

\section{Surgery}

NOTE: Typical instruments used include a scissor, a scalpel, a pair of metallic forceps with straight or curved tips, a tissue retractor tool, several suction wedges and absorbable paper points. The surgery is performed on the left ear.

1. Put the mouse on its right side. Avoid undue torsional stress on the cervical vertebrae. Make sure to keep the body straight to keep the airways open.

2. Cut the fur behind the left ear with a scissor (or shave it with a shaver) to expose the skin. Sterilize the skin with $70 \%$ ethanol solution and betadine (povidone/iodine).

3. Under microscopic magnification (16x), make a $1-1.5 \mathrm{~cm}$ post-auricular incision with the scalpel.

4. Switch to higher microscopic magnification $(25 x)$.

5. Perform blunt dissection through the subcutaneous fat layer, which can be of variable thickness, with forceps. NOTE: Be careful when dissecting as the external jugular vein traverses this area. Damage to this structure can cause excessive bleeding.

6. Retract the sternocleidomastoid muscle to reveal the tympanic bulla periosteum. Use the facial nerve as a key anatomical landmark to aid identification of the auditory bulla. The facial nerve wraps around the posterior/dorsal edge of the sternocleidomastoid muscle and runs rostrally along the ear canal towards the pinna. Gently place the self-retaining retractor tool in the incision to ease access to the bulla (Figure 4).

7. Remove the tissue overlying the medio-dorsal area of the bulla to allow clear visualization of the ridge between the bulla and the mastoid process.

8. Gently rotate a $30 \mathrm{G}$ needle to pierce the bulla and make a hole (bullostomy) on the posterior-superior side of the ridge (the bone is thinner on this side). Alternatively, use a dental surgical drill.

NOTE: This and the following steps can be done with even higher microscopic magnification (40x) if preferred. Also, change the position of the microscope if needed. It is important to maximize the surgical view of the middle ear space.

9. Widen the bullostomy by pinching small bone pieces using fine tipped forceps to expose the middle ear cavity. Extend the bullostomy dorsally towards the mastoid process until the round window niche is clear of overlying bone. The stapedial artery, a branch of the internal carotid artery, runs ventral to the round window niche.

1. Be careful not to damage the vessel as excessive bleeding may be fatal. Small bleeds can be stopped by pressing a small piece of spongostan in the inner ear cavity.

2. Extend the bullostomy toward the anterior-superior direction to visualize the stapes, the middle ear bone connected to the oval window.

10. Remove the stapes with forceps to expose the oval window.

\section{Round Window Application of Ototoxic Agent}

1. Gently perforate the round window and oval window membranes using a blunted $30 \mathrm{G}$ needle. Check that perilymph runs out.

2. Slowly perfuse $0.05 \%$ weight/volume neomycin dissolved in PBS (adjusted to $\mathrm{pH} 7.4$ ) through the oval window. Liquid should flush out of the round window. Repeat the same procedure on the round window. Be careful not to damage the window bone structures with the needle used to perfuse.

3. Place a small piece $\left(1 \mathrm{~mm}^{2}\right)$ of spongostan soaked in neomycin within the round window and oval window niche.

4. Remove the retractor tool, close the incision and wait $30 \mathrm{~min}$.

\section{Post-deafening Acoustic ABR}

1. Record aABR in a similar way as before deafening (Steps 2.2 to 2.8 ) (Figure 2b, Figure $\mathbf{3}$ ).

\section{Insertion of $\mathrm{Cl}$ Electrode Array}

NOTE: The intracochlear electrode array consists of four platinum bands $(\varnothing 0.2 \mathrm{~mm})$ with platinum/iridum parylene insulated wire shielded in a silicone tube (Figure 5).

1. Place the retractor tool in the incision to re-access the bulla.

2. Insert the electrode array into the round window (scala tympani) at a depth where the $4^{\text {th }}$ platinum ring is located just inside the round window. This gives an insertion depth of $\sim 2 \mathrm{~mm}$, corresponding to an intracochlear position at $\sim 30 \mathrm{kHz}{ }^{16}$.

3. Coil the lead wire inside the bulla and glue the wire to the tissue above the bulla. Coiling the wire helps to keep the array in place throughout the experiment.

4. Carefully remove the retractor and close the insertion with tissue glue.

5. Make a small incision $(0.5 \mathrm{~mm})$ in the neck perpendicular to the line between where the active and reference ABR electrodes will be using a tissue scissor. Place the platinum ground ball in the subcutaneous pocket and close the small incision with tissue glue (Figure 6).

6. Connect the electrode array board to the Animal Stimulator Platform. 


\section{Electric Auditory Brainstem Response (eABR)}

NOTE: An Animal Stimulator Platform (ASP) is used to electrically stimulate the electrode array. Other current sources and software systems can be used.

1. Place the ABR electrodes as before (Steps 2.3 to 2.5) (Figure 6).

2. Open the ASP software and define the electric pulse stimulation paradigm. We use a charge-balanced biphasic pulses with $50 \mu \mathrm{s} /$ phase and $10 \mu$ s interphase gap presented at 23.3 pulses per second (pps). The electric stimulation is delivered in monopolar electrode configuration with increasing current levels. A total of 400 responses are averaged at each current level.

3. Present the electric pulse trains and record the evoked eABR response continuously via the TDT headstage, pre-amplifier and auditory processor.

4. Plot and analyze the eABR data via a custom-made matlab script (Figure 7). The script and an example of a recording are provided in the Supplementary.

\section{End of Experiment}

1. At the end of the experiment, euthanize the animal according to institutional guidelines.

2. Carefully open the incision and remove the implant.

3. Ultra-sonicate the electrode array in distilled water for $10 \mathrm{~min}$ to remove tissue debris.

NOTE: The implant can be reused several times if the electrodes are intact and properly conducting. To check this, measure the impedance of the electrodes with a multimeter when the array is dry.

4. Store the electrode array in a dry place.

\section{Representative Results}

The purpose of this study was to describe a reliable model for acute $\mathrm{Cl}$ stimulation in the deafened mouse. Pre- and post-surgical hearing thresholds served as a functional readout of the deafening procedure. Topical application of $0.05 \%$ neomycin in the oval and round window significantly increased click-evoked hearing thresholds by $46 \mathrm{~dB} \pm 6$ (pre- vs post-neomycin: $30.0 \mathrm{~dB} \pm 3.8 \mathrm{vs} 75.7 \mathrm{~dB} \pm 3.7, p=0.0003$, paired t-test, $n=7$ ) (Figure 3). The mouse-sized electrode array was hereafter inserted into the round window (Figure 4, Figure 5). Electric simulation of an intracochlear electrode could reliably generate eABR activity. (Figure 7). In some cases, $\mathrm{Cl}$ stimulation activated the facial nerve and produced a high amplitude wave with either short or long latency (Figure 8A and Figure 8B, respectively). The short latency response was characterized by a rapid amplification of wave IV around $3 \mathrm{~ms}$ and is likely to be a direct response of the facial nerve. The long latency response appeared around 5-6 ms and is likely to be a non-auditory muscle (myogenic) response evoked indirectly by the facial nerve. Facial nerve responses are rarely reported in animal studies in literature but is a well-known complication in human Cl users ${ }^{17,18,19}$. In Figure 8, facial nerve stimulation appeared at relatively medium current levels (150-200 $\mu \mathrm{A})$ and in two different animals. In other cases, both responses could appear in the same animal at very high current levels (not shown). We recommend limiting the current level to levels below the appearance of facial nerve stimulation.

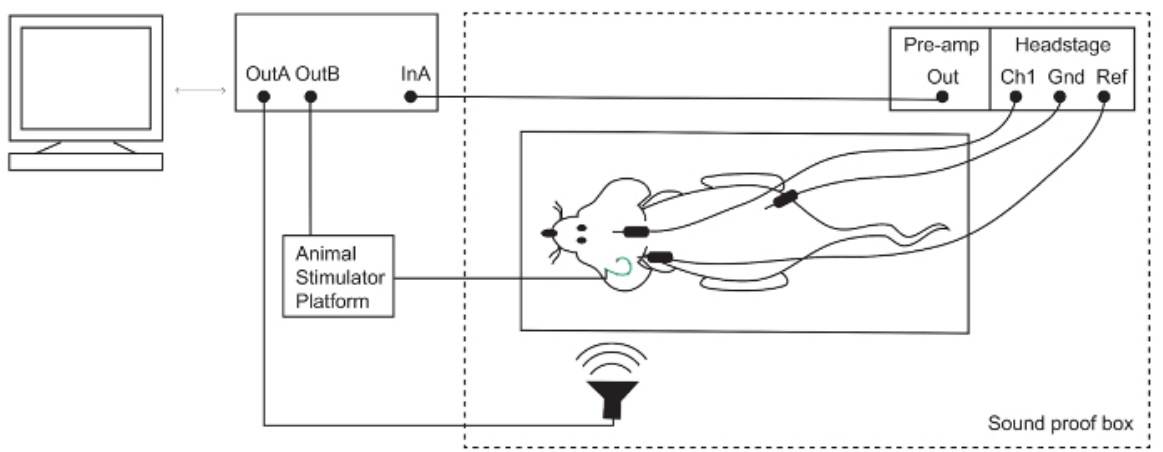

Figure 1: Auditory Brainstem Response (ABR) setup. Subdermal electrodes are placed at the vertex (active/channel 1 [Ch1]), behind the ipsilateral ear (reference [Ref]) and at the hind leg (ground [Gnd]) of the anesthetized mouse. Electrode signals are amplified and then recorded by a TDT system. Acoustic and electric stimulation are presented via a microphone and an Animal Stimulator Platform, respectively. Please click here to view a larger version of this figure. 

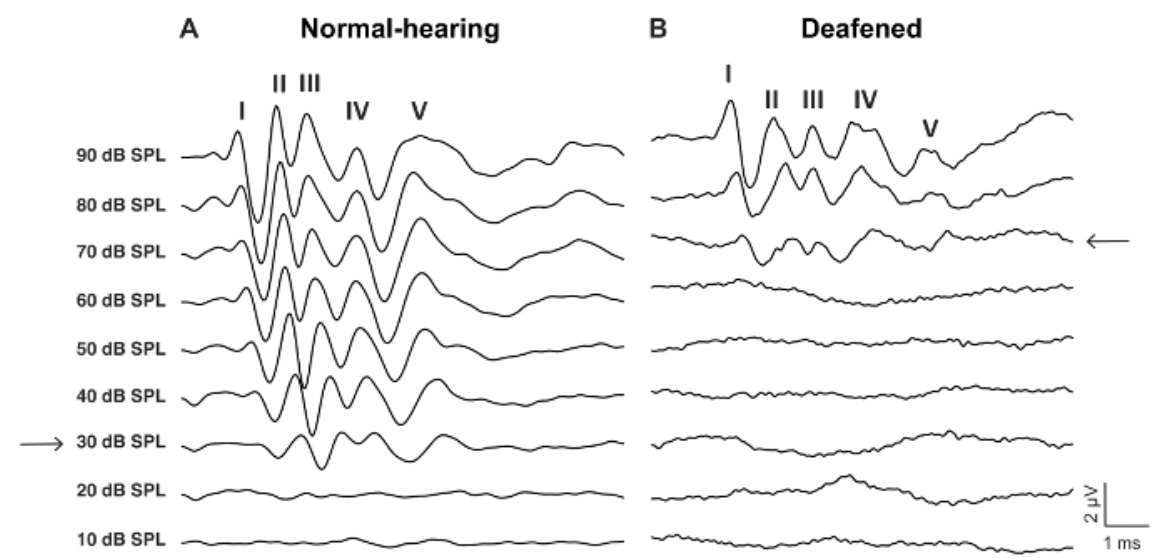

Figure 2: Representative aABR waves to click stimulation from a wild-type mouse before and after deafening with $0.05 \%$ neomycin. (A) The normal-hearing aABR pattern is characterized by waves labelled I-V and a low hearing threshold, here $30 \mathrm{~dB}$ SPL (arrow). (B) The deafened aABR pattern shows an increased hearing threshold, here $70 \mathrm{~dB}$ SPL (arrow). The waves have a longer latency and more temporal jitter. Please click here to view a larger version of this figure.

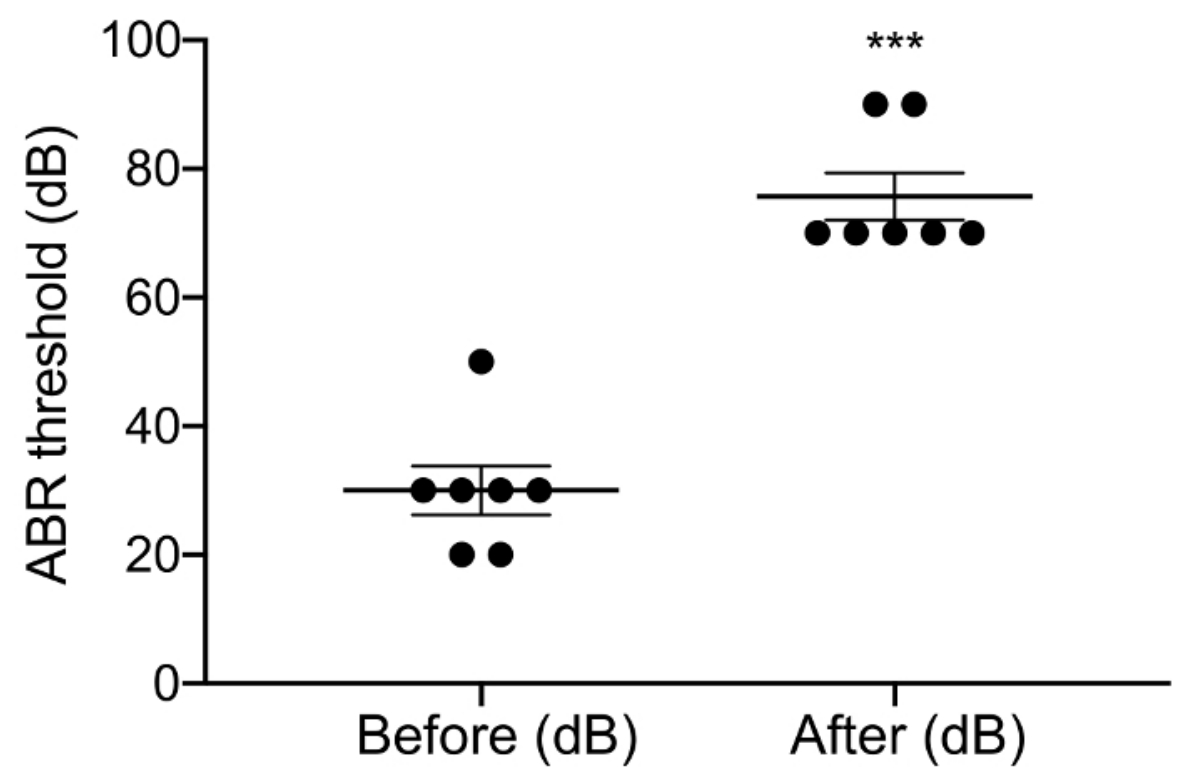

Figure 3: aABR threshold before and after deafening. Application of neomycin significantly increased aABR thresholds by $46 \mathrm{~dB} \pm 6$. Pre- vs post-neomycin: $30.0 \mathrm{~dB} \pm 3.8 \mathrm{vs} 75.7 \mathrm{~dB} \pm 3.7, \mathrm{p}=0.0003$, paired t-test, $\mathrm{n}=7$. Errors are standard error of the means. Please click here to view a larger version of this figure.
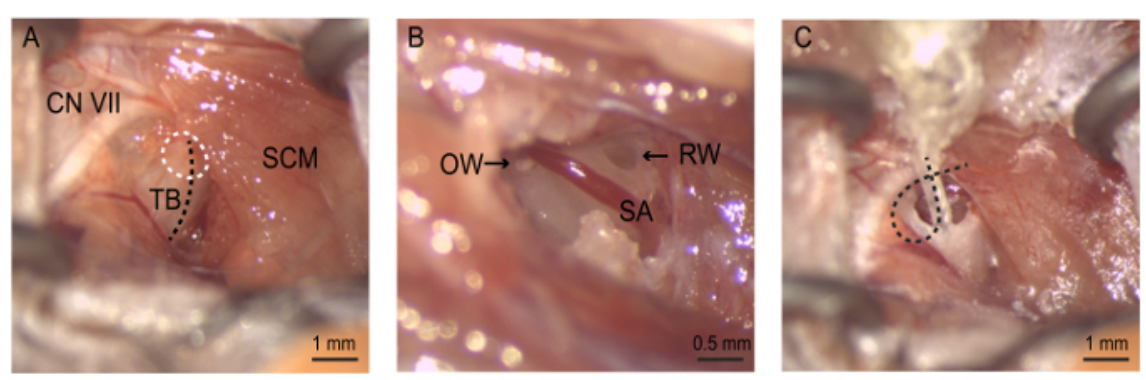

Figure 4: The surgery. (A) Exposure to the auditory bulla. The bullostomy is performed (white dotted circle) along the ridge on the tympanic bulla (black dotted line). (B) The bullostomy allows visualization of the round window, stapedial artery, and oval window. Neomycin is gently flushed through first the oval window, then the round window. (C) The electrode array is inserted until the $4^{\text {th }}$ electrode is located just inside the round window niche. The electrode wire is coiled inside the bulla to keep the array in place before the incision is closed. $\mathrm{CN}$ VII $=\mathrm{cranial}$ nerve VII (facial nerve), OW = oval window, $\mathrm{RW}=$ round window, $\mathrm{SA}=$ stapedial artery, $\mathrm{SCM}=$ sternocleidomastoid muscle, $\mathrm{TB}=$ tympanic bulla. Please click here to view a larger version of this figure. 

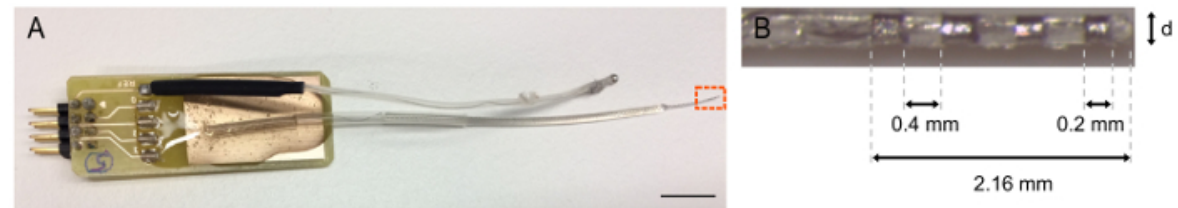

Figure 5: The mouse cochlear implant. (A) The intracochlear electrode array consists of four platinum bands spaced at a $0.4 \mathrm{~mm}$ interval with a diameter $d$ : 0 [tip] $(d=0.21), 1(d=0.23), 2(d=0.25), 3(d=0.27)$. The width of each electrode is $0.2 \mathrm{~mm}$. The four platinum/iridium (90/10) parylene insulated wires are shielded in a silicone tube. (B) Magnification of the electrode array tip (red dotted square). The electrode array and a platinum reference ball are connected to a print board. Scale bar $=1 \mathrm{~mm}$. Please click here to view a larger version of this figure.

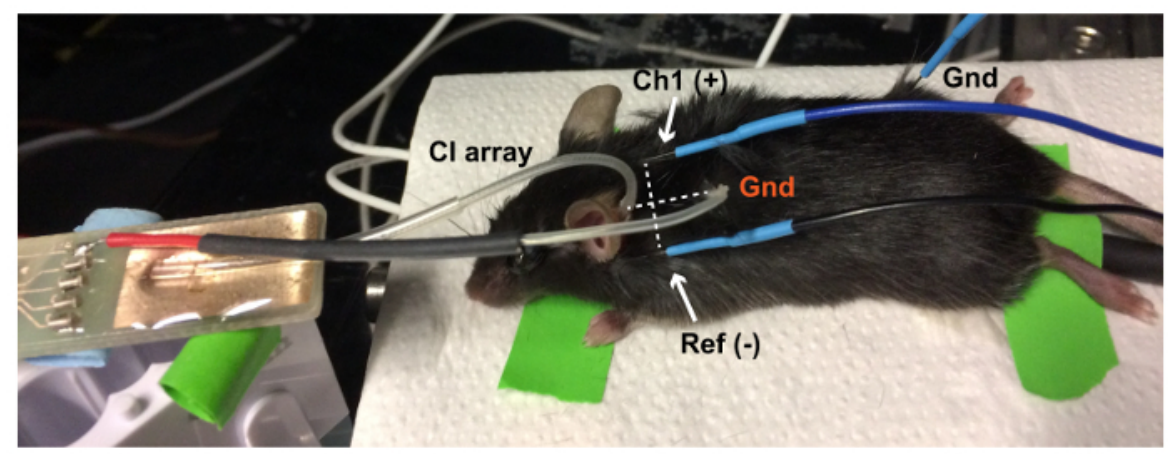

Figure 6: Electrically-evoked ABR (eABR) setup. The $\mathrm{Cl}$ platinum ground ball (Gnd, red) is placed in a subcutaneous pocket in the neck of the mouse. The line between active $(\mathrm{Ch} 1(+)$ at the vertex) and reference (Ref $(-)$ at the ipsilateral ear) ABR electrodes is perpendicular to the line between the electrode array and the ground in order to obtain the best eABR response. The eABR ground electrode (Gnd, black) is placed in the hind leg. Please click here to view a larger version of this figure. 


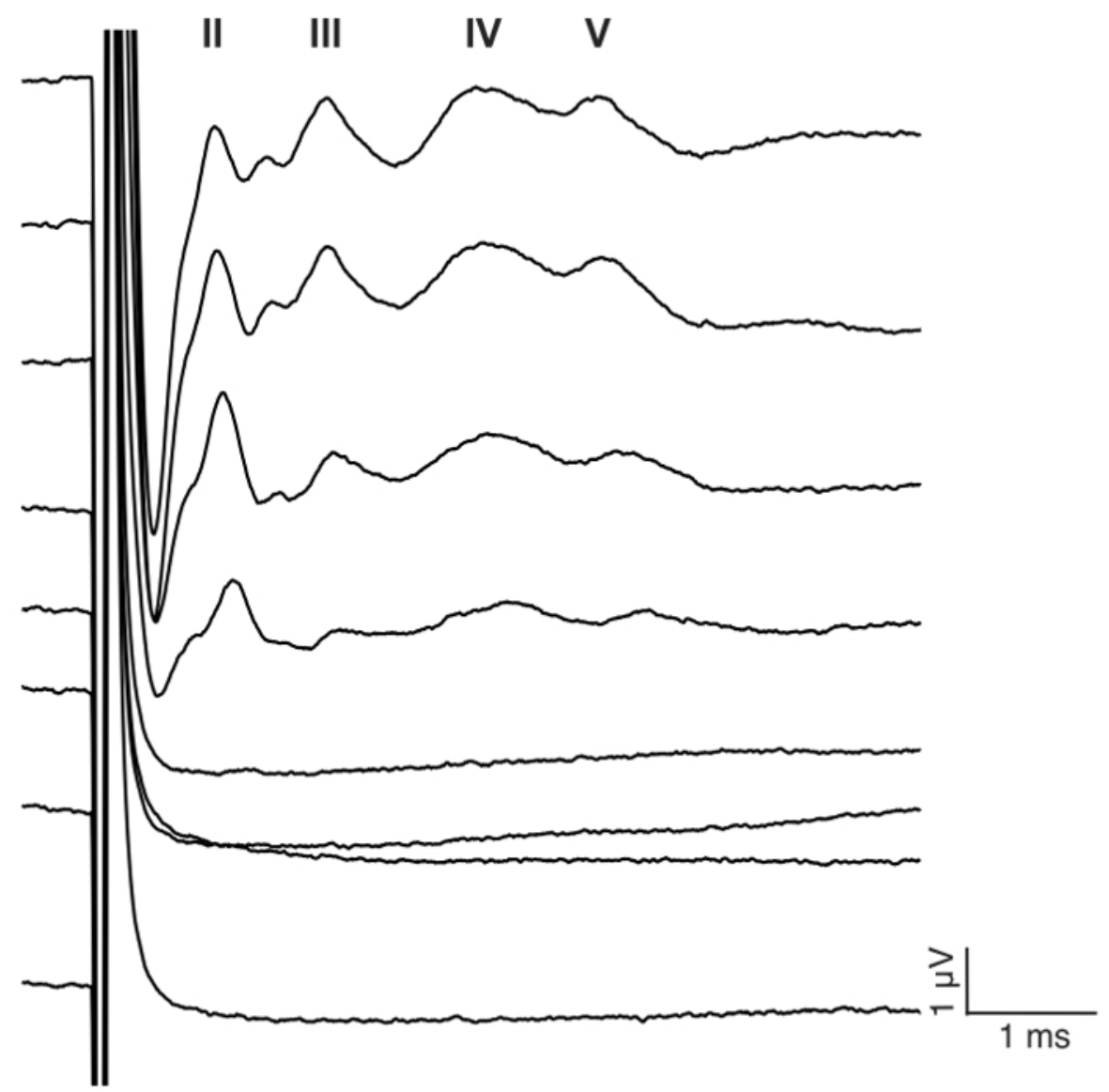

Figure 7: Representative eABR waves to $\mathrm{Cl}$ stimulation in a deafened mouse. A biphasic pulse train is presented to electrode \#1 in monopolar configuration at 23.3 pulses per second (pps) with 400 repetitions. Stimuli level $0-175 \mu A$ is shown in $25 \mu \mathrm{A}$ steps (see stimulation details in Step 7.2). Roman numerals denote eABR wave number. The wave amplitudes and latency increase and decrease, respectively, with increasing current level. In this example, wave II appeared around $1 \mathrm{~ms}$, wave III around $2 \mathrm{~ms}$, wave IV around $3 \mathrm{~ms}$, wave V around $4 \mathrm{~ms}$. Please click here to view a larger version of this figure.

A

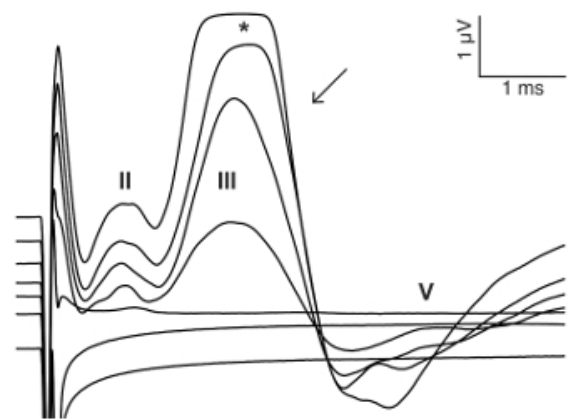

B

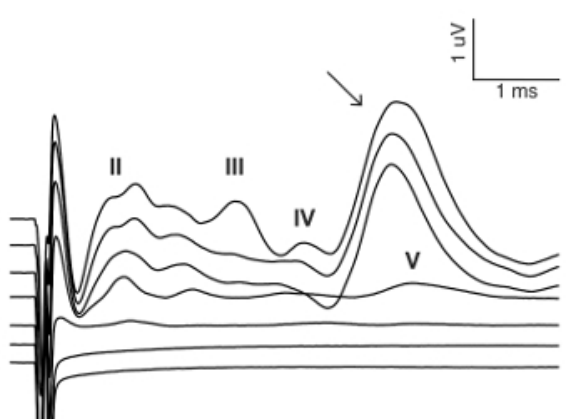

Figure 8: Example of facial nerve stimulation. In some cases, $\mathrm{Cl}$ stimulation can activate the facial nerve and evoke a direct response with short latency (A) (arrow) or indirect response with longer latency (B) (arrow). The examples shown are from two Cl-implanted animals stimulated with a biphasic pulse train using $0-300 \mu \mathrm{A}$ in $50 \mu \mathrm{A}$ steps (see stimulation details in Step 7.2). Roman numerals denote eABR wave numbers. * denotes clipping of the eABR wave due to saturation of the amplifier. Please click here to view a larger version of this figure.

\section{Discussion}

This manuscript describes the surgical approach for acute deafening and cochlear implantation in the mouse, as well as the functional assessment of $\mathrm{Cl}$ stimulation with auditory brainstem response. Although the mouse cochlea is small and the surgery challenging, the $\mathrm{Cl}$ mouse model is feasible and serves as a valuable tool in auditory research.

The stapedial artery is present in the middle ear of the mouse. The artery enters the bulla posterior-medially, and runs inferiorly to the round window niche and then superiorly to the oval window niche. In the initial development of the mouse model, we experienced fatal intra-operative 
bleeding following trauma to the stapedial artery, mainly while accessing the bulla. As a consequence, we adapted a more limited approach and accessed the bulla in smaller, refined dissection steps. No further complications due to bleeding were thereafter observed. Despite the fact that stapedial artery cauterization has no significant effect on hearing threshold or number of spiral ganglion neurons in mice ${ }^{5}$, in our opinion, it is unnecessary so long as great care and attention are taken during the surgery. We suggest taking the time needed to develop fine psychomotor skills and reach technical proficiency. The average time from initial incision to closure around the implanted electrode array is typically $1-1.5 \mathrm{~h}$.

The described acute $\mathrm{Cl}$ surgery in mice is similar to the "ventral" procedure and round window insertion used in other rodents, including rats and gerbils $\mathrm{s}^{20,21,22}$. Other rodent studies have used the "dorsal approach" with a basal turn cochleostomy instead of a round window insertion, avoiding the SA entirely and inserting the array more deeply ${ }^{6,23,24}$. The implantation of a chronic stimulation assembly in mice follows the same steps as described in this protocol with the addition of a Dacron mesh to fix the implant and postoperative care ${ }^{5}$.

The main technical challenges when performing $\mathrm{Cl}$ surgeries in mice are the small size of the cochlea compared to the cochlea of rats and gerbils, and the presence of a large SA. The SA is also present in rats but not in gerbils. In addition, since mice are smaller than rats and gerbils, they are more vulnerable to surgical procedures.

To eliminate electrophonic responses in eABR recordings and to mimic hair cell loss found in most $\mathrm{Cl}$ users, we deafened the animals before $\mathrm{Cl}$ insertion. Mice are difficult to deafen ototoxically in vivo ${ }^{25}$ because the systemic concentrations of aminoglycosides required to cause ototoxicity has a narrow dose window: lower doses given over several days results in no hair cell loss whereas a single injection of a higher dose can be lethal $^{26}$. Also, susceptibility to aminoglycosides is strain dependent ${ }^{26}$. However, it has been shown that a single dose of aminoglycosides in combination with a loop diuretic can produce excessive outer hair cell loss in CBA/CaJ mice without fatal consequences ${ }^{27}$. Delayed inner hair cell death was reported in half of all cochleae examined ${ }^{27}$.

In this manuscript, we used topical application of the aminoglycosides neomycin inspired by the protocol recently established for C57BL/6 mice 5 . Acute application of neomycin significantly increased the click-evoked hearing threshold by $46 \mathrm{~dB} \pm 6.1$. Although this increase is larger than the $35 \mathrm{~dB}$ increase reported by Irving et al. (pre- vs post-surgery: $41.6 \mathrm{~dB} \pm 3.3 \mathrm{vs} 76.6 \mathrm{~dB} \pm 4.4, \mathrm{p}=0.02, \mathrm{n}=3$ ) , we achieved the same postdeafening threshold $(75.7 \mathrm{~dB} \pm 3.7 \mathrm{vs} 76.6 \mathrm{~dB} \pm 4.4) .0 .05 \%$ neomycin is thought to cause a partial loss of hearing, mainly by rapid outer hair cell death, as inner hair cell loss takes longer to occur ${ }^{27}$. It is therefore possible that electrophonic response, which is generated both by inner and outer hair cells $4,8,9,10,11,12$, is only partially eliminated in deafened animals with residual hearing. Even though $0.05 \%$ (weight/volume) neomycin does not decrease the number of spiral ganglion neurons 4 weeks post-deafening ${ }^{5}$, it is yet unknown if neomycin in our acute setup affects the auditory nerve fibers or promotes synaptopathy (loss of synapses between inner hair cells and type I auditory nerve fibers). Another uncertainty is that the topical neomycin treatment may not produce a uniform distribution of hair cell loss along the length of the cochlea. Future studies are required to answer these questions.

In summary, the growing number of genetic models for human deafness and the biochemical tools available make the mouse an attractive animal model for auditory research, including the field of Cls.

\section{Disclosures}

No competing financial interests. The authors have nothing to disclose.

\section{Acknowledgements}

The authors would like to thank Pierre Stahl, Oticon Medical, Nice, France, for providing the Animal Stimulation Platform and advice on stimulation paradigms, and James B. Fallon and Andrew K. Wise from Bionics Institute, Melbourne, Australia, for surgical advice. This work was supported by a grant from the Swiss National Science Foundation (ERC transfer grant to T.R.B.).

\section{References}

1. The-Ear-Foundation. Cochelar Implants Update. (2018).

2. Fallon, J. B., Irvine, D. R. F., \& Shepherd, R. K. Cochlear Implants and Brain Plasticity. Hearing Research. 238 (1-2), $110-111$ (2008).

3. Pfingst, B. E. et al. Neurotrophin Gene Therapy in Deafened Ears with Cochlear Implants: Long-term Effects on Nerve Survival and Functional Measures. Journal of the Association for Research in Otolaryngology. 18 (6), 731-750 (2017).

4. Miller, C. A. et al. Electrical excitation of the acoustically sensitive auditory nerve: single-fiber responses to electric pulse trains. Journal of the Association for Research in Otolaryngology. 7 (3), 195-210 (2006).

5. Irving, S. et al. Cochlear implantation for chronic electrical stimulation in the mouse. Hearing Research. 306 37-45 (2013).

6. Soken, H. et al. Mouse cochleostomy: a minimally invasive dorsal approach for modeling cochlear implantation. Laryngoscope. 123 (12), E109-115 (2013).

7. Mistry, N., Nolan, L. S., Saeed, S. R., Forge, A., \& Taylor, R. R. Cochlear implantation in the mouse via the round window: effects of array insertion. Hearing Research. 312 81-90 (2014).

8. Hartmann, R., Topp, G., \& Klinke, R. Discharge patterns of cat primary auditory fibers with electrical stimulation of the cochlea. Hearing Research. 13 (1), 47-62 (1984).

9. Sato, M., Baumhoff, P., \& Kral, A. Cochlear Implant Stimulation of a Hearing Ear Generates Separate Electrophonic and Electroneural Responses. The Journal of Neuroscience. 36 (1), 54-64 (2016).

10. Pfingst, B. E., Spelman, F. A., \& Sutton, D. Operating ranges for cochlear implants. Annals of Otology, Rhinology \& Laryngology. 89 (2) (1980).

11. Miller, C. A., Hu, N., Zhang, F., Robinson, B. K., \& Abbas, P. J. Changes across time in the temporal responses of auditory nerve fibers stimulated by electric pulse trains. Journal of the Association for Research in Otolaryngology. 9 (1), 122-137 (2008). 
12. Shepherd, R. K., \& Javel, E. Electrical stimulation of the auditory nerve. I. Correlation of physiological responses with cochlear status. Hearing Research. 108 (1-2), 112-144 (1997).

13. Akil, O., Oursler, A. E., Fan, K., \& Lustig, L. R. Mouse Auditory Brainstem Response Testing. Bio Protocol. 6 (6) (2016).

14. Willott, J. F. Measurement of the auditory brainstem response (ABR) to study auditory sensitivity in mice. Current Protocols in Neuroscience. Chapter 8 (Unit 8.21B) (2006).

15. TDT. ABR User Guide: A Guide to ABR Testing with the System 3 RZ6. Updated August 2017. Florida, USA. (2017).

16. Muller, M., von Hunerbein, K., Hoidis, S., \& Smolders, J. W. A physiological place-frequency map of the cochlea in the CBA/J mouse. Hearing Research. 202 (1-2), 63-73 (2005).

17. Cushing, S. L., Papsin, B. C., \& Gordon, K. A. Incidence and characteristics of facial nerve stimulation in children with cochlear implants. Laryngoscope. 116 (10), 1787-1791 (2006).

18. Berrettini, S., Vito, D. A., Bruschini, L., Passetti, S., \& Forli, F. Facial nerve stimulation after cochlear implantation: our experience. Acta Otorhinolaryngologica Italica. 31 (1), 11-16 (2011).

19. Hu, H., Kollmeier, B., \& Dietz, M. Reduction of stimulation coherent artifacts in electrically evoked auditory brainstem responses. Biomedical Signal Processing and Control. 21 74-81 (2015).

20. Wiegner, A., Wright, C. G., \& Vollmer, M. Multichannel cochlear implant for selective neuronal activation and chronic use in the free-moving Mongolian gerbil. Journal of Neuroscience Methods. 273 40-54 (2016).

21. Hessel, H. et al. Meriones unguiculatus (Gerbil) as an animal model for the ontogenetic cochlear implant research. American Journal of Otolaryngology. 18 (S21) (1997).

22. Pinilla, M., Ramirez-Camacho, R., Jorge, E., Trinidad, A., \& Vergara, J. Ventral approach to the rat middle ear for otologic research. Otolaryngology Head Neck Surgery. 124 (5), 515-517 (2001).

23. King, J., Shehu, I., Roland, J. T., Jr., Svirsky, M. A., \& Froemke, R. C. A physiological and behavioral system for hearing restoration with cochlear implants. Journal of Neurophysiology. 116 (2), 844-858 (2016).

24. Lu, W., Xu, J., \& Shepherd, R. K. Cochlear implantation in rats: a new surgical approach. Hearing Research. 205 (1-2), 115-122 (2005).

25. Poirrier, A. L. et al. Ototoxic drugs: difference in sensitivity between mice and guinea pigs. Toxicology Letters. 193 (1), $41-49$ (2010).

26. Wu, W. J. et al. Aminoglycoside ototoxicity in adult CBA, C57BL and BALB mice and the Sprague-Dawley rat. Hearing Research. 158 (1-2), 165-178 (2001).

27. Taylor, R. R., Nevill, G., \& Forge, A. Rapid hair cell loss: a mouse model for cochlear lesions. Journal of the Association for Research in Otolaryngology. 9 (1), 44-64 (2008). 